\title{
Visual impairment in severe and profound sensorineural deafness
}

\author{
I M Armitage, J P Burke, J T Buffin
}

\begin{abstract}
The frequency of reversible and irreversible visual impairment was determined in children with severe and profound sensorineural deafness, as subnormal vision can adversely affect their educational and social development. Eighty three of 87 such children attending an audiology service were examined to assess the incidence and severity of visual impairment. Each child underwent a detailed ophthalmic assessment. The criteria for visual impairment were visual acuity $<6 / 9$ Snellen or equivalent and/or abnormal binocular vision. Forty five had a normal ophthalmic examination $(54 \cdot 2 \%)$. Twenty nine had visual impairment (34.9\%) and nine had ophthalmological abnormalities that did not interfere with vision $(10.9 \%)$.

A higher proportion of children with risk factors for visual pathology demonstrated visual impairment than those in whom there were no risk factors. None the less, $44 \%$ of visual impairment was among patients without risk factors. The results underline the need to examine all children with severe and profound sensorineural deafness soon after diagnosis and indicate that children with multiple handicaps have a greater likelihood of visual impairment (11 of 14 cases).

(Arch Dis Child 1995; 73: 53-56)
\end{abstract}

Keywords: screening, sensorineural deafness, visual impairment.

Much of a child's learning is dependent upon listening to speech and other environmental sounds and most of the learning that is necessary for speech development takes place during the first two years of life. ${ }^{1}$ A deaf child is therefore dependent on vision for communication and learning. Hearing and visually impaired children are significantly more debilitated, being less able to lip read, less cooperative, and less capable of manual tasks than hearing impaired children with normal vision. ${ }^{2}$

Previous studies have reported the frequency of visual impairment among the entire hearing impaired population, ${ }^{3-11}$ but failure to specify the degree of hearing impairment and the differences in the definition of visual impairment made comparisons between these studies difficult.

The present study was set up to determine the frequency of visual morbidity among a subgroup of UK children with severe and pro- found sensorineural hearing impairment and to make recommendations for their ophthalmological assessment.

\section{Methods}

The study sample consisted of all 87 children from the Sheffield area with severe $(>70$ $\mathrm{dBHL}$ ) and profound ( $>90 \mathrm{dBHL}$ ) sensorineural hearing impairment. The hearing loss was assessed in the Department of Paediatric Communication using an audiogram, and assessed hearing thresholds at octave frequencies of $500,1000,2000$, and $4000 \mathrm{~Hz}$. In all cases the better ear average was used to categorise the degree of hearing impairment. The sample included all children in the Sheffield area with severe and profound sensorineural hearing impairment, regardless of whether they had a diagnosed or suspected visual problem.

The criteria for visual impairment were visual acuity of $<6 / 9$ Snellens or equivalent and/or abnormal stereoacuity. Information on audiological, past medical, birth, and family histories together with history of maternal drug use during pregnancy were obtained from the parents and supplemented from the hospital records. Assessment of ocular status comprised visual acuity testing, assessment of the patient's ability to fuse the visual input from each eye (binocular vision) and measurement of stereopsis (binocular vision), cycloplegic refraction, and ophthalmological examination. The ideal standard for visual acuity testing was 6/9 Snellen or equivalent, using Sheridan Gardiner singles or linear, Kay pictures, Cardiff cards, or preferential looking tests. Visual acuity was assessed uniocularly and binocularly. Each visual acuity test was used only on the age group of child for which it was designed and the results were not extrapolated into standard Snellen equivalents. Binocular vision assessment was made using the cover test, Bagolini glasses or Worth's lights, prism fusion range, and stereoacuity tests. Stereoacuity of 100 seconds of arc or better enables fine depth perception and was considered to be normal. Stereoacuity worse than 100 seconds was recorded as reduced and where stereoacuity was not demonstrable, absent stereopsis was recorded (table). Ocular motility was assessed in nine positions of gaze, testing both ductions and versions. Convergence and optokinetic nystagmus were recorded. Cycloplegic refraction using cyclopentalate hydrochloride $1 \%$ or atropine sulphate $1 \%$ was performed and all children underwent slit lamp examination, direct and indirect ophthalmoscopy. 
Summary of the number of children with ophthalmological abnormalities and these as a percentage of the total sample (83 children)

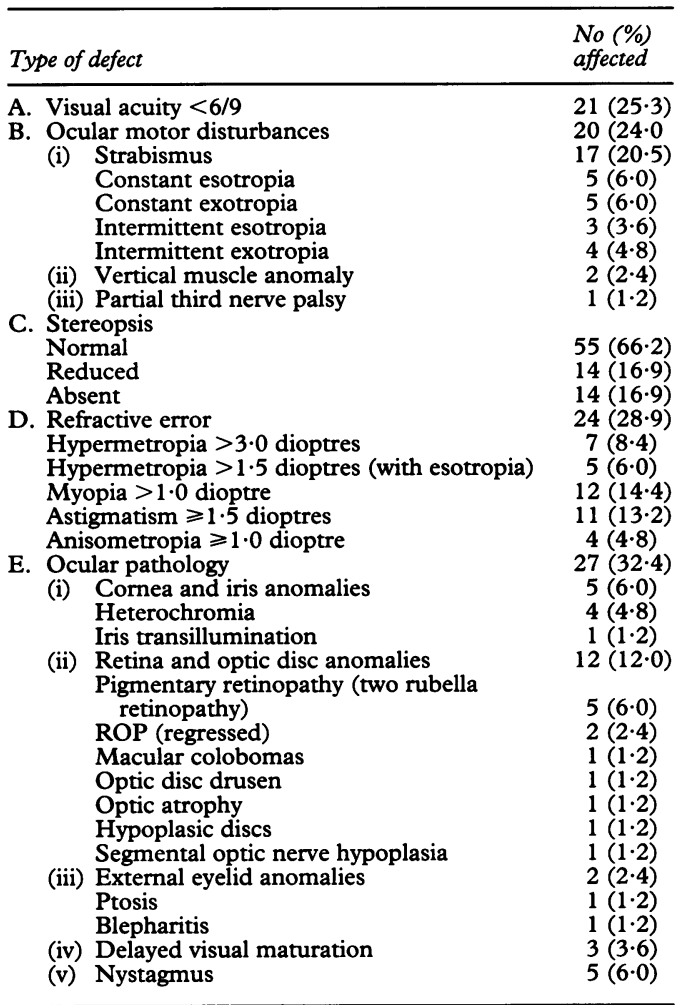

$\mathrm{ROP}=$ retinopathy of prematurity.

Electrodiagnostic tests were not performed routinely.

The refractive errors considered to be significant were hypermetropia of $\geqslant 1.5$ dioptres in the presence of esotropia (allowance was made for working distance only) and $>3.0$ dioptres in the absence of manifest strabismus (with allowance for cycloplegia and working distance), myopia of $>1.0$ dioptre, astigmatism of $\geqslant 1.5$ dioptres, and anisometropia of $\geqslant 1 \cdot 0$ dioptre.

The aetiology of the hearing impairment was categorised as known congenital, known acquired, or unknown. Congenital hearing impairment was attributable to a congenital syndrome, intrauterine infection - for example, rubella, cytomegalovirus, or toxoplasmosis - or where there was a positive family history of hearing impairment and no other apparent cause. Acquired hearing impairment was due to, for example, prematurity or perinatal complications, meningitis, and other acquired infection.

The data obtained from this study sample were compared with a study which was accepted as accurately reflecting the pattern of visual impairment in a normal population. Coleman analysed 3623 children with normal hearing, between kindergarten and sixth grade, with criteria similar to those of the present study. ${ }^{12}$ Statistical analysis was carried out on the data. The $x^{2}$ test was employed for the analysis of specified groups within the hearing impaired sample and $95 \%$ confidence limits were applied when comparing the findings of the present study against existing data.

\section{Results}

Eighty three of the 87 hearing impaired children in the sample attended for examination $(95.4 \%)$. The age range was from 16 months to 16 years at the time of examination. The mean age was 9.5 years and the median age was 8.5 years. There were 41 males and 42 females. Forty six children had severe and 37 profound sensorineural hearing impairment.

Forty five children $(54 \cdot 2 \%)$ had a normal ophthalmological examination. Twenty nine children $(34.9 \%)$ met the criteria for visual impairment. Of these 15 had severe and 14 had profound sensorineural hearing loss.

Twenty one cases had visual acuity of worse than $6 / 9$ Snellen or equivalent at initial examination. Of these children $42.9 \%$ (nine of 21 cases) had visual acuity of $6 / 12$ or $6 / 18,33.3 \%$ (seven of 21 ) had visual acuity of $6 / 24$ to $6 / 60$, and $23.8 \%$ (five of 21 ) had visual acuity of less than $6 / 60$. Fifty five children $(66 \cdot 2 \%)$ had normal stereopsis. In 14 cases (16.9\%) stereopsis was reduced and in 14 cases $(16.9 \%)$ it was absent.

A disturbance of ocular motility was present in 20 cases $(24.0 \%)$. Seventeen cases had concomitant horizontal strabismus $(20.5 \%)$, and three had vertical muscle anomalies. Ocular pathology (excluding refractive error and strabismus) was present in 27 children $(32.4 \%)$ and contributed to visual impairment in 18 cases.

In total, 38 children ( $45 \cdot 8 \%$ ) had visually significant abnormalities and/or visually insignificant ophthalmological anomalies, with 13 having one and 25 having two or more defects. Nine of the 38 cases had an isolated anomaly not interfering with visual acuity, but whose presence was useful in diagnosing the aetiology of the hearing impairment.

Fifty four children $(65 \cdot 1 \%)$ had a single handicap (that is, severe or profound sensorineural deafness), $18(21 \cdot 7 \%)$ had a dual handicap where defects of hearing and vision were present, and $14(16.9 \%)$ had multiple handicaps. The latter included hearing impairment and combinations of visual impairment, cerebral palsy, epilepsy, neurodevelopmental delay, and microcephaly. Three children with multiple handicaps did not demonstrate visual impairment.

The aetiology of the hearing impairment was categorised as congenital in 44 cases, acquired 13 cases, and of unknown origin in 26 cases. Sensorineural deafness in association with a syndrome was present in 14 children (three Waardenburg's syndrome, one Down's, one Weaver's, one DIDMOAD (diabetes insipidus, diabetes mellitus, optic atrophy, deafness), one Donaldson's, one Zellweger's, four with rubella, and two with multisystem abnormalities in association with deafness. Of these, 10 had visually significant ophthalmic findings and the remainder had incidental ophthalmic abnormalities.

Of the 21 children with visual acuity of $<6 / 9$ Snellen or equivalent, nine had irreversible visual loss, two had partially reversible visual loss associated with regressed retinopathy of prematurity, and visual loss was completely 
reversible in 10 cases and restored to normal with the provision of the correct refraction. Absent or reduced stereopsis was not reversible in 19 cases. In the 14 children where stereopsis was absent manifest strabismus was elicited in all cases and in five cases where stereopsis was permanently reduced but not absent, convergent squint, microsquints, rubella retinopathy, and bilateral superior oblique weakness were the causes. In the remaining nine cases with reduced stereopsis the prescription of corrective spectacle lenses restored it to normal levels.

Certain risk factors identify neonates at risk for visual impairment. In this study prematurity or perinatal complications, exposure to ototoxic medications, rubella and cytomegalovirus infection, neurodevelopmental handicaps, and syndromes with known hearing and ophthalmic complications, were considered significant risk factors for visual impairment. Children with hearing impairment were divided into two groups: those in whom there was no reason to anticipate ocular abnormalities and/or visual impairment and those in whom the history indicated an increased risk. Twenty four children had risk factors for visual impairment and of these 16 had significant visual impairment $(66 \cdot 6 \%)$. The remaining 59 children had no risk factors for visual impairment. Of these, 13 had visual impairment $(22.0 \%)$. These data show that a significantly higher proportion of visual impairment occurred when the history indicated an increased risk for visual impairment $\left(\chi^{2}=14 \cdot 87, \mathrm{df}=1, \mathrm{p}<0.05\right)$. However, of the total sample almost half the visual impairment (13 of 29 cases) was found in children without risk factors.

The aetiology of hearing impairment was categorised as congenital (44 cases), acquired (13 cases), and unknown (26 cases). In the congenital category, 19 children had visual impairment, while four of the acquired group and six of those with unknown aetiology had visual impairment. The slight difference in the incidence of visual impairment among the congenital and acquired hearing impaired groups is not statistically significant $\left(\chi^{2}=0.58, \mathrm{df}=1\right.$, $\mathrm{p}>0.05$ ).

Fifty four of the 83 children in the sample had not undergone ophthalmic examination before this study. Fourteen of the 29 children with visual impairment were newly diagnosed. Of these, eight had significant visual impairment which was fully reversible in seven children and irreversible in one child. Before this survey 15 children had a diagnosis of visual impairment. Of these children, those with multiple handicaps had their visual impairment diagnosed as soon as the other impairments. However, the age of diagnosis of visual impairment in the others was delayed a mean of three years after the diagnosis of severe or profound sensorineural hearing impairment.

\section{Discussion}

Epidemiological studies on the frequency of visual impairment among the entire hearing impaired population exist, ${ }^{3-11}$ but are often difficult to interpret because of their failure to use recognised definitions for a variety of visual functions. More than $45.0 \%$ of hearing impaired children in this study had one or more significant ocular abnormalities. Of these 38 cases, 29 had visual impairment and nine had anomalies which were not visually significant. Our criteria for visual impairment were practicable because they did not exaggerate the frequency of ocular problems and allowed comparison to be made with two other studies. Rogers et al reported $43 \%$ in their series $(n=360$, aged 6 to 21 years) had significant ocular findings, ${ }^{10}$ while Regenbogen and Godel noted $45.3 \%$ of children with ocular abnormalities which interfered with vision ( $n=150$, aged 4 to 14 years). ${ }^{8}$ The incidence of visual impairment in the total hearing impaired group in our study was significantly higher than that of $17.5 \%$ found in a normal school population. ${ }^{12}$ However, when considering the proportion of children in the study with a dual handicap of hearing and visual impairment $(21 \cdot 7 \%)$, the incidence of visual impairment is much closer to the reported incidence in the normal school population. While it may be argued that the presence of severe and profound sensorineural hearing loss in children without additional handicaps may not be an indicator for the ophthalmological screening of these children, it does suggest that a significantly higher proportion of children with these levels of hearing loss do have additional impairments, which includes visual impairment. We therefore consider that these children should undergo routine ophthalmic assessment.

At initial ophthalmic assessment, subnormal visual acuity was present in 21 of the study children $(25.3 \%)$, binocular vision was absent in 14 cases, and reduced in a further 14 children (16.9\%). After treatment visual acuity loss in nine children and reduced binocular vision in five children remained irreversible. All children in whom visual acuity loss was irreversible had multiple handicaps and significant ocular pathology.

Unlike some studies, 57 well controlled latent strabismus was not considered clinically significant. The incidence of manifest strabismus was $20.5 \%$ and this was up to four times higher than previously reported. ${ }^{10}$ The incidence of manifest strabismus in a normal population has been cited at $1 \cdot 8 \%{ }^{12}$ and $4 \cdot 6 \% .13$ When tested at $95 \%$ confidence limits its incidence is significantly greater than even the highest estimate in the normal population.

Refractive errors were the most frequently encountered ocular abnormalities and this confirms the findings of previous studies. ${ }^{811}$ Significant visual impairment was present in $83.3 \%$ of children with uncorrected refractive errors, which emphasises the importance of correction. The incidence of astigmatism in this study was significantly higher than in the normal population while the incidence of hypermetropia and myopia were not.

The retina and cochlea develop from the same embryonic layers during the sixth and 
seventh weeks after conception. In the present study $16.9 \%$ of children had oculoauditory syndromes and $71.4 \%$ (10 of 14 ) had visual impairment. This is similar to the $16.7 \%$ incidence of oculoauditory syndromes in Regenbogen and Godel's paper. ${ }^{8}$ No patient in this study had ophthalmic symptoms or clinical signs indicative of Usher's syndrome which are minimal in the first decade of life. This contrasts with the incidence of 1.1 to $1.9 \%$ reported when electrodiagnosis was performed routinely. ${ }^{10}$

The early detection of sensorineural hearing loss in infancy is important but difficult to accomplish and often delayed. ${ }^{14}$ Consequently the formulation of an effective ophthalmological screening policy is equally difficult to achieve, as significant visual problems may have already developed by the time sensorineural hearing impairment is detected. The mean period of delay of diagnosis of visual impairment for children in this study was three years after the diagnosis of the hearing impairment in children without multiple handicaps. It would seem more prudent to maximise visual function in severely hearing impaired children without undue delay, because of the dependence they have upon the visual system for acquiring communication and language skills. $^{25}$

In this study the incidence of visual impairment fell into two groups: those in whom there was no reason to anticipate visual impairment (13 of 59 cases) and those in whom the history indicated an increased risk (16 of 24 cases). Although a higher proportion of children in the 'at risk' group had visual impairment than in the 'no risk' group, almost half of the total visual impairment in the sample was found in children who did not demonstrate risk factors. Similarly, the likelihood of visual impairment was independent of the aetiology of the hearing impairment (congenital or acquired). It was noteworthy that six of 29 cases $(20 \cdot 7 \%)$ had newly diagnosed visual impairment and benefited from early diagnosis.

Severely hearing impaired children are especially dependent upon vision for communication and learning and they should be given every opportunity to maximise their visual potential at the earliest age possible. As we have been unable to identify a subgroup of hearing impaired children within this study as having a higher risk of visual impairment, we would recommend that all children with severe and profound sensorineural hearing impairment undergo ophthalmological assessment soon after diagnosis of their hearing impairment. Follow up ophthalmic and orthoptic examinations should be individualised and routine because of the changing nature of refractive errors and the possibility of the later onset of symptomatic retinal degeneration in certain children. ${ }^{9}$

1 Chaurasia MK, Geddes NK. An analysis of the aetiology of early childhood deafness. Clin Otolaryngol 1991; 16: $280-4$.

2 Suchman RG. Visual impairment among deaf children Arch Ophthalmol 1967; 77: 18-21.

3 Braly KW. A study of defective vision among deaf children. Am Ann Deaf 1938; 83: 192-3.

4 Stockwell E. Visual defects in deaf children. Arch Ophthalmol 1952; 48: 428.

5 Luhr JP, Dayton F. combining clinical and educational services. Am Ann Deaf 1971; 116: 566-8.

6 Alexander JC. Ocular abnormalities among congenitally deaf children. Can $\mathcal{f}$ Ophthalmol 1973; 8: 438-42.

7 Mohindra I. Vision profile of deaf students. American Fournal of Optometry 1976; 53: 412-9.

8 Regenbogen L, Godel V. Ocular deficiencies in deaf child ren. $\mathcal{F}$ Pediatr Ophthalmol Strabismus 1985; 22: 231-3.

9 Fillman RD, Leguire LE, Rogers GL, Bremer DL, Fellows RR. Screening for vision problems including Usher's syndrome among hearing-impaired students. Am Ann Deaf 1987; 132: 194-8.

10 Rogers GL, Allman RD, Bremer DL, Leguire LE. Screening of school-aged hearing impaired children. f Pediatr Ophthalmol Strabismus 1988; 25: 230-2.

11 Leguire LE, Fillman MA, Fishman DR, Brewer DL, Rogers GL. A prospective study of ocular abnormalities in hearing impaired and deaf students. Ear Nose Throat $\mathcal{F} 1992$; 71: 643-51.

12 Coleman HM. An analysis of an entire school population. 7 Am Optom Assoc 1970; 41: 341-7.

13 Rantanen A, Tommila V. Prevalence of strabismus in Finland. Acta Ophthalmol (Copenh) 1971; 49: 506-7.

14 Wild NJ, Sheppard S, Smithells RW, Holzell H. Delayed detection of congenital hearing loss in high risk infants. BMF 1990; 301: 903-5. 